\title{
USB-Powered Coated Blade Spray lon Source for On-Site Testing Using Transportable Mass Spectrometry
}

\author{
Josha Jager, Arjen Gerssen, Janusz Pawliszyn, Saskia S. Sterk, Michel W. F. Nielen, \\ and Marco H. Blokland*
}

Cite This: J. Am. Soc. Mass Spectrom. 2020, 31, 2243-2249

Read Online

\section{ACCESS | Lill Metrics \& More | 回 Article Recommendations ｜（） Supporting Information}

ABSTRACT: On-site testing in food analysis using mass spectrometry (MS) requires miniaturization of vacuum systems, mass analyzers, sample cleanup, and ionization sources. In this study, a simple coated blade spray (CBS) ion source was developed that enables high voltage generation on the blade by ubiquitous certified (micro-)USB On-The-Go devices like smartphones, tablets, and power banks. CBS is capable of performing both analyte enrichment by solid-phase microextraction (SPME) material coated on the metal substrate and direct-spray ionization. The USB-CBS device was used on two

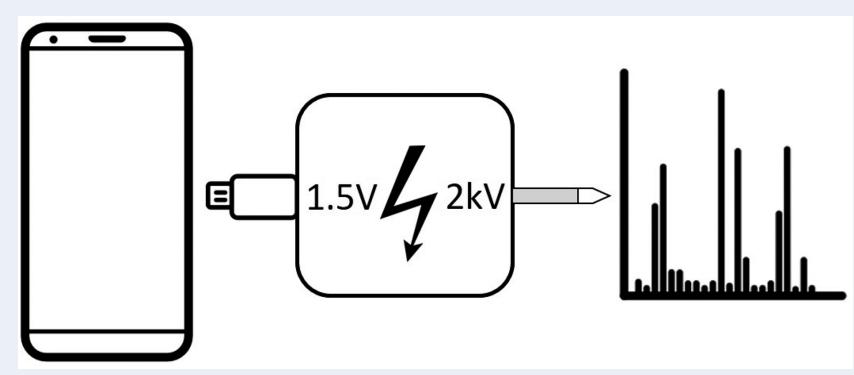
different MS systems, a transportable single-quadrupole and a benchtop triple-quadrupole tandem MS. Various characteristics of the USB-CBS device, including high voltage generation and angular positioning, were studied. The potential of the newly developed device for food safety applications is demonstrated by banned and regulated veterinary drugs such as $\beta$-agonists and sulfonamide antibiotics, covering a wide range of molecular weights and polarities. The results highlight the potential of the developed, simplified, inexpensive (less than 10 USD), and universal vendor-independent USB-powered CBS ion source coupled with MS(/MS) systems for semiquantitative applications, in laboratories, and in future on-site food quality and safety testing. Apart from that, most likely onsite environmental, biomedical, and forensic testing will also benefit from this USB-CBS instrumental development that is compatible with any atmospheric inlet MS system.

\section{INTRODUCTION}

Mass spectrometry (MS) and tandem (MS/MS) applications are popular in quantitative analysis because of their sensitivity, selectivity, and wide applicability. With recent (academic) developments, portable miniaturized mass spectrometers are becoming more prevalent and will eventually find their way from research laboratories to control laboratories to further onsite or at-line measurements. ${ }^{1-11}$ However, besides the portability of the mass analyzer, another prerequisite for onsite measurements is the simplification of sample preparation and subsequent ionization for introducing food contaminant extracts into the mass analyzer. Electrospray-like ionization methods, directly from substrates, showed promising applicability for on-site food safety analysis. ${ }^{12}$ Minimal sample preparation effort, absence of gas supply, direct analysis of trace amounts deposited on surfaces, and a wide range of available and inexpensive substrates ${ }^{13,14}$ are also a prerequisite for the success of a future on-site application.

Coated blade spray (CBS) is, in theory, an ideal compromise between sample cleanup and direct ionization. ${ }^{15,16} \mathrm{CBS}$ blades are geometrically sharp-tipped stainless-steel strips that are coated with a biocompatible SPME sorbent material. ${ }^{15}$ The merging of SPME coating and metal support for direct spray ionization contributes to both the reduction of organic solvent used for extraction and the omission of gas supply or vulnerable laser to assist in desorption ionization. CBS allows for the direct extraction of compounds from liquids, which could be easily performed on-site while adding crucial selectivity for a wide range of analytes by selecting blades with dedicated SPME sorbent phase coatings. ${ }^{17}$ There are several fully validated methods for analyzing food contaminants using CBS published. ${ }^{16,18}$

Future end-users of portable direct ionization MS systems for on-site analysis will be most likely inspectors. Apart from training in basic skills, on-site MS analysis should as much as possible be cost-efficient, user-friendly, safe, and self-explanatory by design. One way of enabling more user-friendly operation is combining a direct ionization source with a ubiquitous device such as a smartphone or tablet. ${ }^{19}$ Using smartphones would enable future operation by a simplified smartphone or tablet app, so the end-user can be supported

Received: August 14, 2020

Revised: September 30, 2020

Accepted: October 12, 2020

Published: October 21, 2020 
and even remotely guided during operation. ${ }^{20}$ However, commercial ion sources generally lack such flexibility, are rather complicated and expensive. Moreover, future (trans)portable MS systems may suffer from insufficient HV-supply for robust operation of direct ionization techniques such as CBS.

Anticipating on future simplified portable MS and MS/MS instrumentation, we took up the challenge to build a USB powered ion source using very inexpensive components. This study demonstrates a simple, quick, and cheap universal vendor-independent, but robust, ion source that can be used for CBS and can be applied to any atmospheric pressure inlet MS. The designed and constructed device generates a high voltage (HV) from USB supplied low voltage, with the capability of utilizing any certified micro-USB On-The-Go (OTG) mobile telephone as a power supply. Apart from the characterization of the USB-CBS ion source, application to different model compounds and other food safety-related examples is described.

\section{EXPERIMENTAL SECTION}

Chemicals. Methanol (MeOH) and Milli-Q water (all ULC/MS grade) were purchased from Actu-All (Oss, The Netherlands). Analytical standards of clenbuterol, fenoterol, isoxsuprine, procaterol, salbutamol, salmeterol, terbutaline, ritodrine, metaproterenol, ractopamine, sulfadiazine, sulfamerazine, sulfamethizole, sulfamethoxazole, sulfamethoxypyridazine, sulfapyridine, sulfaquinoxaline, sulfathiazole, sulfamoxole, sulfacetamide, sulfaphenazole, [D-Lys3]-GHRP-6, and brombuterol- $d_{9}$ were purchased from Sigma-Aldrich (St. Louis, MO). Sulfadimidine and sulfachlorpyridazine were provided by Riedel de Haen (Seelze, Germany). Ostarine and andarine were purchased from Selleck Chemicals (Houston, TX), and recombinant bovine somatotropin (rbST) was obtained from the National Hormone \& Peptide Program (NHPP) of Dr. Parlow (Torrance, CA). Brombuterol, cimaterol, cimbuterol, clencyclohexerol, clenhexyl, clenpenterol, clenproperol, mabuterol, mapenterol, tulobuterol, chlorbrombuterol, hydroxymethylclenbuterol, and sulfapyridine- ${ }^{13} \mathrm{C}_{6}$, sulfamerazine $-{ }^{13} \mathrm{C}_{6}$, sulfamethizole- ${ }^{13} \mathrm{C}_{6}$, sulfamethoxypyridazine- $d_{3}$, sulfachlorpyridazine- ${ }^{13} \mathrm{C}_{6}$, sulfisoxazole $-{ }^{13} \mathrm{C}_{6}$, and sulfaquinoxaline- ${ }^{13} \mathrm{C}_{6}$ were purchased from Witega (Berlin, Germany). Carbuterol, reproterol, zilpaterol, clenbuterol- $d_{6}$, salbutamol- $d_{6}$, ractopamine- $d_{5}$, mabuterol- $d_{9}$, cimbuterol- $d_{9}$, cimaterol- $d_{7}$, clenproperol- $d_{7}$, isoxsuprine- $d_{5}$ and mapenterol- $d_{11}$ were kindly provided by BVL, EURL for B-agonist-type substances (Berlin, Germany). Zilpaterol- $d_{7}$, terbutaline- $d_{9}$, fenoterol- $d_{6}$, reproterol- $d_{4}$, clencyclohexerol- $d_{10}$, and carbuterol- $d_{9}$ were purchased from Toronto Research (Toronto, ON, Canada). Bovine urine samples were obtained from multiple bovine animals of different age and gender from routine monitoring programs in The Netherlands. Bovine milk samples were from laboratory stock and predetermined blank for sulfonamide antibiotics.

Instruments and Consumables. The blades used for CBS were coated with either Bondelut Certify (Agilent, Amstelveen, The Netherlands) or Oasis HLB (Waters, Milford, MA) stationary-phase material at Waterloo University (Waterloo, ON, Canada). Inexpensive $\mathrm{HV}$ generators for positive (\$5) and negative ionization (\$2) and a USB adjustable output transformer board $(\$ 2)$ were purchased at the Alibaba Group (Hangzhou, China). The USB-provided input voltage was maintained at $1.5 \mathrm{~V}$ for all positive ionization experiments and $2.0 \mathrm{~V}$ for all negative ionization experiments.
Coated blades were mounted via a (toothless crocodile) clamp connected to the HV output wire. The ground wire was connected to a grounded part of the mass spectrometer. An example of the USB ionization setup, using a micro-USB smartphone is given in Figure 1. The mass spectrometers used

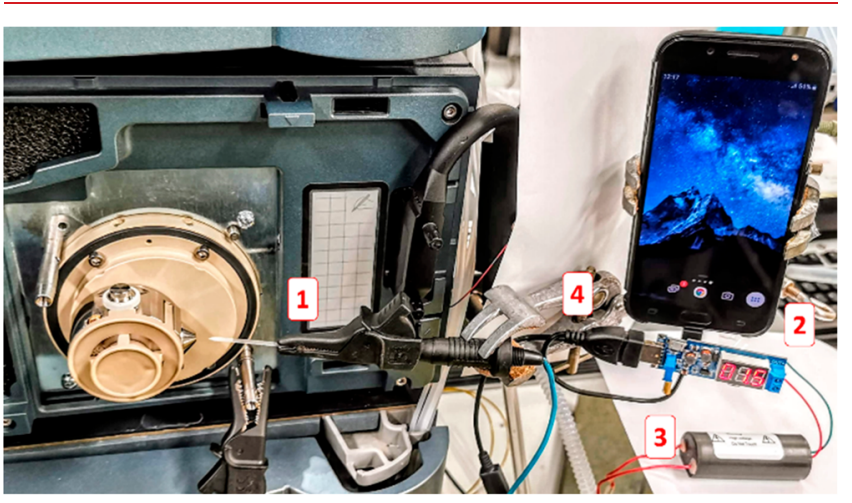

Figure 1. Instrumental setup of an OTG micro-USB phone connected to an adjustable DC/DC USB transformer board coupled to the positive HV generator connected to the coated blade via a toothless crocodile clamp for CBS ionization: (1) crocodile clamp fixating a blade, (2) DC/DC USB transformer board, (3) HV generator module, (4) micro-USB power supply.

were a benchtop model Quattro Premier XE triple quadrupole tandem MS system (Waters) and a model Acquity QDa (Waters) transportable single quadrupole MS detector. To perform CBS analysis, both the Premier XE and QDa were slightly modified. At the Premier XE, the ESI source housing and probe were removed and interlocks were bypassed by a dummy plug (MS Vision, Almere, The Netherlands). The QDa was slightly modified by removing the ESI source housing and changing the instrument settings, according to Trimpin et al. $^{21}$

CBS Methodology. The CBS method was adopted from Tascon et al. ${ }^{22}$ Coated blades were preconditioned before sampling using $\mathrm{MeOH} /$ water $(50: 50 \mathrm{v} / \mathrm{v})$ and vortex mixing for $30 \mathrm{~s}(\mathrm{~s})$ at $650 \mathrm{rpm}(\mathrm{rpm})$. After preconditioning, sampling of blades was performed by placing the blade in an aliquot of $300 \mu \mathrm{L}$ of standards, blanks, or fortified urine samples and vortexing at $650 \mathrm{rpm}$ for $60 \mathrm{~s}$. Some of the sample matrix contaminants were removed by a 2 -fold wash with $300 \mu \mathrm{L}$ of water, discarding the liquid in between the washing steps, and vortexing during $10 \mathrm{~s}$. Blades were dried in the open-air for approximately $5 \mathrm{~min}$. Desorption and ionization were performed using $10 \mu \mathrm{L}$ of $\mathrm{MeOH}$, followed by the application of the selected HV for approximately $20 \mathrm{~s}$.

Benchtop MS/MS Measurements. Source and desolvation temperatures were set at 100 and $20{ }^{\circ} \mathrm{C}$ respectively. Desolvation and cone gas flows were both set to $0 \mathrm{~L} / \mathrm{h}$, and collision-induced dissociation (CID) was performed utilizing a $0.18 \mathrm{~mL} / \mathrm{min}$ argon (purity $\geq 99,998 \%$ ) gas flow. For each sulfonamide antibiotic two product-ions were measured, the cone voltage, collision energy and $\mathrm{m} / z$ values are given in Table S1. Instrument control and analysis of MS data were carried out using MassLynx v4.1 software (Waters).

Transportable Single-Quadrupole MS Measurements. Initial QDa MS data were obtained in full scan positive ion mode at a cone voltage of $15 \mathrm{~V}$ and source temperature of $150{ }^{\circ} \mathrm{C}$. The theoretical monoisotopic mass of each precursor ion was measured in selected ion recording 
(SIR) data acquisition mode. Instrument control and analysis of MS data were performed using MassLynx v4.1 software.

Data Processing. For the quantitative determination of the analytes of interest, the area under the specific ion signal in the smoothed reconstructed ion chromatogram (RIC) was used. Smoothing of the RIC was done by applying the SavitzkyGolay algorithm using a window size of 2 . The ratios between the area of the RICs of the analyte and its internal standard were expressed as a response factor. Calibration curves corresponding to individual analytes were constructed by plotting the response factor versus the analyte concentration. Linearities were determined using correlation coefficients based on least-squares linear regression.

Single-Day Initial Validation. Method linearity was determined using the correlation coefficients of the constructed calibration curves. Calibration curves were constructed in triplicate by multianalyte fortified matrices in the concentration range $0-200 \mu \mathrm{g} / \mathrm{kg}(n=5)$. The limit of detection (LOD) of individual analytes was defined as the first concentration in the calibration curve with a response factor higher than the previous lower calibration concentration used.

\section{RESULTS AND DISCUSSION}

Design and Characterization of the USB-CBS Ion Source. For creating a USB-powered CBS ion source, a positive direct current to direct current (DC/DC) HV generator module was developed composed of a cored transformer and multiple capacitors for HV generation. Details of the USB-HV source can be found elsewhere. ${ }^{19}$

Various input voltages and distances of the CBS tip to the inlet of the MS were evaluated in order to obtain a stable spray formation without discharge. The input voltages were in the range of $1.0 \mathrm{~V}$ up to $2.5 \mathrm{~V}$ and distances in the range of $2.0 \mathrm{~mm}$ to $20 \mathrm{~mm}$. The optimum input voltage was $1.5 \mathrm{~V}$ and a distance of $8.0 \mathrm{~mm}$. These initial tests clearly show Taylor cone formation and the associated spray jet (see video S1). However, the spray onset of the USB-CBS device also showed a transient aerosol outburst when the $\mathrm{HV}$ was activated. To determine the origin of the observed aerosol outburst, the USB-CBS was placed in front of an MS and an oscilloscope high-voltage probe measurement was performed. The oscilloscope high-voltage probe enabled the recording of the HV build-up curve of the created USB-CBS device. The recorded curve showed that the maximum output voltage of the created USB-CBS device was approximately 2700 V, i.e., in an appropriate range for electrospray-like coated blade spray (see Figure S1a).

The voltage curve of the conventional MS powered high voltage, set at $1600 \mathrm{~V}$ in the software, was likewise recorded by the oscilloscope (see Figure S1b). A comparison of both HV curves showed remarkable differences in voltage build-up. At the configured USB-CBS device the voltage rapidly increased prior to stabilization, while the MS powered setup showed a much slower HV build-up followed by slope deflection before a final stabilizing at the set value of $1600 \mathrm{~V}$.

Because of the nature of the new voltage generator device, the rapid voltage build-up cannot be avoided. Due to the initial outburst, the obtained MS signal might be initially less stable compared to the slow voltage build-up in conventional, MS system powered, HV supplies. The use of an appropriate internal standard is common practice in CBS applications ${ }^{15,16,22,23}$ and will correct for the HV profile differences, as was verified by analyzing clenbuterol, spiked in an aqueous solution at three concentrations $(5,50$, and $100 \mu \mathrm{g} / \mathrm{L})$ and a corresponding internal standard clenbuterol- $d_{6}(5 \mu \mathrm{g} / \mathrm{L})$, data not shown. The two different methods of HV generation, USBand MS system-powered CBS, demonstrated similar linearities $\left(R^{2}=0.996\right.$ and $\left.R^{2}=0.997\right)$ after correcting with the internal standard.

Angular Blade Positioning to the Mass analyzer. Due to limited access and space for positioning of the CBS device in front of the inlet of different brands of mass spectrometers, angular positioning of the USB-CBS device to the MS inlet would be required. While the significance of angular positioning of CBS techniques relative to the MS inlet has not been reported in the literature, the tolerance to horizontal positioning of CBS for effective ionization in front of the MS inlet has been described. ${ }^{15,24}$ Gómez-Ríos et al. achieved stable high signal intensities for $5 \mathrm{~mm}$ in all directions from the center of the ion-transfer capillary. ${ }^{15}$ Similar tolerances to the positioning have been described for paper spray MS analysis. ${ }^{24}$ Since minor offsets in relative angular positioning of the USBCBS device could have similar effects as small offsets in horizontal placement, the effect on the total ion current (TIC) and the RIC of $50 \mu \mathrm{g} / \mathrm{L}$ clenbuterol and $5 \mu \mathrm{g} / \mathrm{L}$ clenbuterol- $d_{6}$ were investigated. Blades were kept at a similar distance of 8 $\mathrm{mm}$ to the MS inlet and directly pointed at the center of the MS inlet. The straight central positioning of the CBS device in front of the MS inlet is considered as an angular positioning of $180^{\circ}$. The four tested angles relative to the MS inlet are $190^{\circ}$, $210^{\circ}, 225^{\circ}$, and $240^{\circ}$, as depicted in Figure 2.
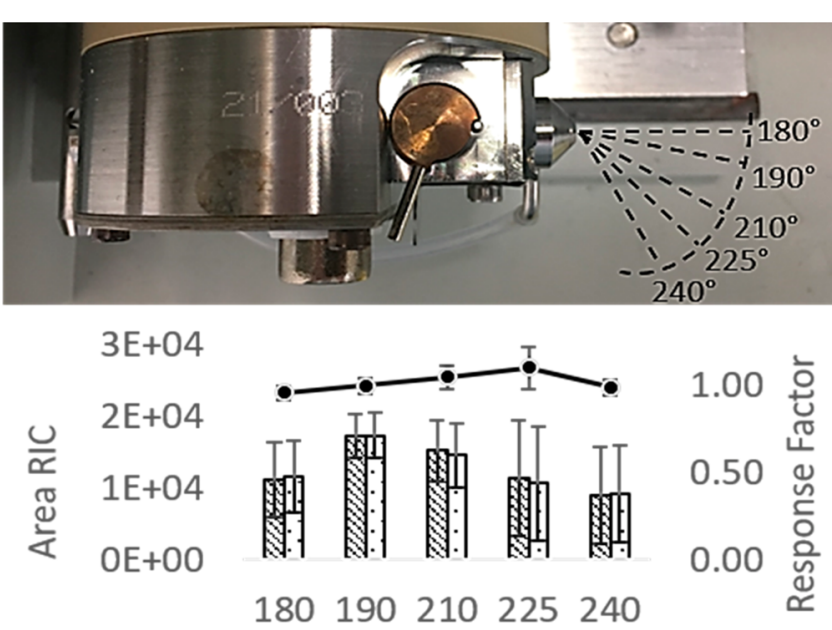

\section{Angle coated blade to MS inlet $\left({ }^{\circ}\right)$}

Figure 2. Top panel: Representation of the conventional $180^{\circ}$ and the four tested angular offsets utilized for characterization of tolerance to angular positioning relative to the MS inlet. Lower panel: Area RICs clenbuterol (diagonal stripes) and clenbuterol- $d_{6}$ (dots) at the different angular offsets, including calculated response factor (solid line) and standard deviations $(n=3)$.

Only a minor effect of lower RICs of clenbuterol was observed at higher angular offsets, as shown in Figure 2. An increase in standard deviation (\% real.SD) is observed by offsets beyond $190^{\circ}$ when not corrected for the added internal standard. In contrast, the use of isotope-labeled internal standard results showed highly reproducible response factors of $0.96,1.00,1.05,1.10$, and 0.99 , respectively. Consequently, the tested angles could all be applied without compromising linearity. To reduce errors caused by manual blade fixation, an 

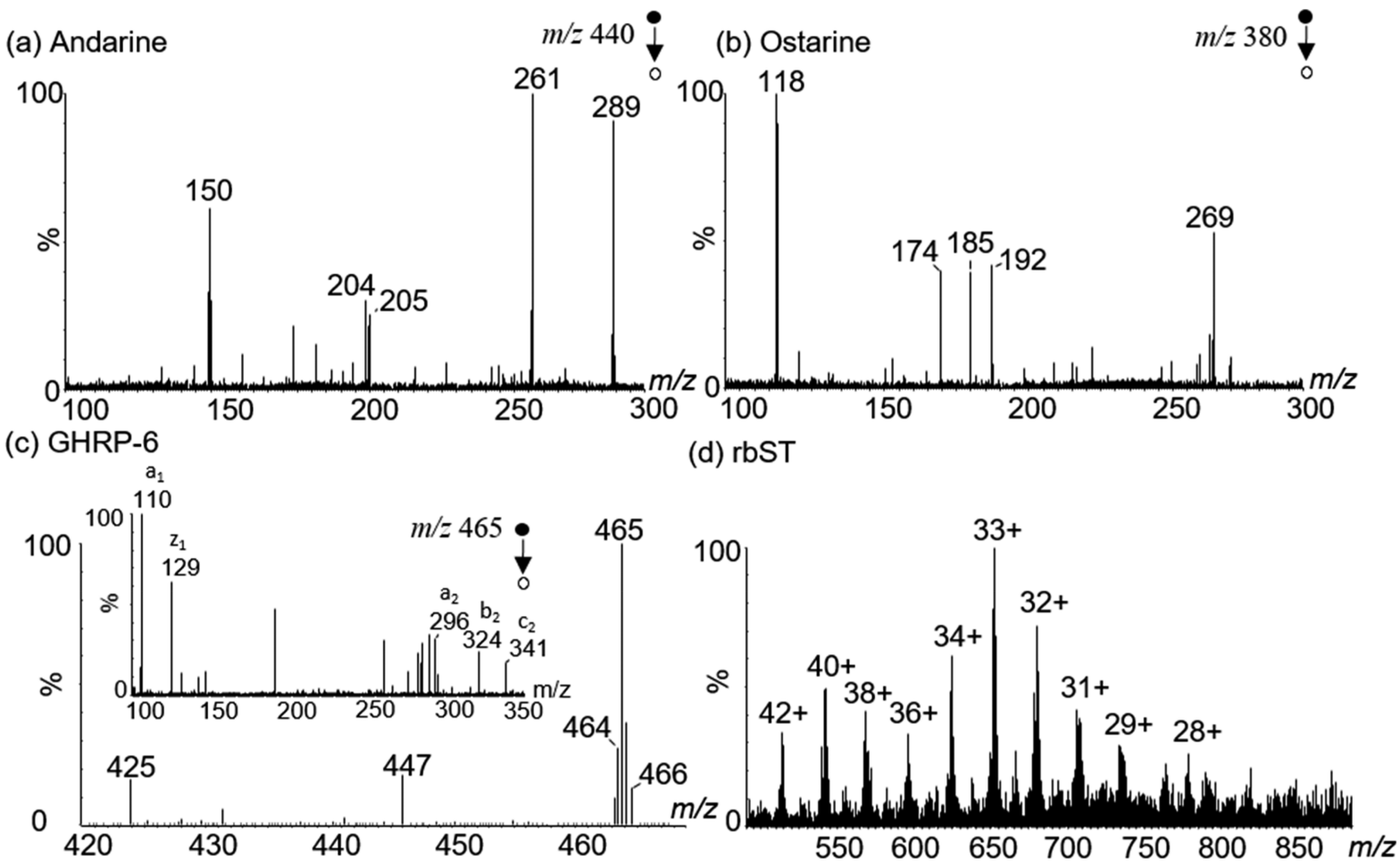

Figure 3. USB-CBS mass spectra of aqueous solutions showing (a) negative ionization mode product ion scan of andarine (50 $\mu \mathrm{g} / \mathrm{L}),(\mathrm{b})$ negative ionization mode product ion scan of ostarine $(50 \mu \mathrm{g} / \mathrm{L}),(\mathrm{c})$ positive ionization mode full and product ion scans of [D-Lys3]-GHRP-6 (1.0 $\mu \mathrm{g} /$ $\mathrm{mL})$, and (d) positive ionization mode full scan of intact $\mathrm{rbST}(30 \mu \mathrm{g} / \mathrm{mL})$.

autosampler such as the previously commercially developed for paper spray may be considered. ${ }^{25}$

Proof of Principle of USB-CBS-Transportable-MS. The developed simplified CBS ion source was combined with a transportable single-quadrupole mass spectrometer system (QDa). This commercial MS system has a small footprint, can be easily transported and is up and running in only 10 min. $^{3}$ For demonstrating the USB-CBS-transportable-MS analysis, $\beta$-agonists were used. ${ }^{26-28}$

The affinity of $\beta$-agonists for Bondelut Certify coated material has been previously described ${ }^{12,29}$ and was expected the same for all the $\beta$-agonists, given the similarities in basicity and amine moieties. ${ }^{15,30}$ The compatibility of the Bondelut blades was confirmed by a full scan mass spectrum of an aqueous solution containing $25 \beta$-agonists on the transportable MS. Out of the MS spectrum, 20 clear signals could be obtained for $22 \beta$-agonists (see Figure S2). The recorded mass spectrum (Figure S2) included two overlapping isobaric signals for clenpenterol $(m / z 291)$ and procaterol $(m / z 291)$, ractopamine $(\mathrm{m} / z 302)$, and isoxsurpine $(\mathrm{m} / z$ 302) due to similar monoisotopic masses that are not separated by the low resolution single-stage quadrupole MS. Signals of reproterol $(\mathrm{m} / z$ 390), brombuterol $(\mathrm{m} / \mathrm{z} 367)$, and zinterol $(\mathrm{m} / z$ 379) could not be detected, in agreement with the reduced sensitivity observed in our LC-MS/MS routine method. To determine the LOD a calibration series of $\beta$-agonists spiked in bovine urine in the range of 2 to $2000 \mu \mathrm{g} / \mathrm{L}$ was analyzed in duplicate in SIR mode using $\mathrm{m} / z$ values of the selected $\beta$ agonists. For clenbuterol, mabuterol, mapenterol, tulobuterol, chlorbrombuterol, hydroxymethylclenbuterol, clenproperol, cimaterol, cimbuterol, ritodrine, salmeterol, clenhexyl, and procaterol the LOD was in the range of 20 to $50 \mu \mathrm{g} / \mathrm{L}$. For clenpenterol, isoxsuprine, ractopamine, clencyclohexerol, sal- butamol, zilpaterol, fenoterol, carbuterol, and metaproterenol the range was 100 to $2000 \mu \mathrm{g} / \mathrm{L}$. While signals of $22 \beta$-agonists at higher concentrations in aqueous solutions and in urine could be observed by low-resolution single-quadrupole MS, this MS system provides insufficient selectivity and sensitivity at the low regulatory limits for $\beta$-agonists in bovine urine.

USB-CBS-MS(/MS) Analysis of SARMs, GHRPs, and rBST. To gain more selectivity and sensitivity, required to compensate for the lack of chromatographic separation prior to ionization as demonstrated by the $B$-agonists experiment, a tandem mass analyzer is needed. ${ }^{31}$ Therefore, further experiments were performed on an outdated benchtop triplequadrupole MS/MS system, assuming to match the (limited) sensitivity of future truly portable triple-quadrupole mass spectrometers. Model analytes with different physicochemical properties were selected to demonstrate the capability of USB-CBS for different molecular weights: the selective androgen receptor modulators (SARMs) ostarine and andarine (380 and $441 \mathrm{Da}$ ), a growth hormone-releasing peptide (GHRP, $930 \mathrm{Da}$ ) and recombinant growth hormone somatotropin protein $(\mathrm{rbST}, 21.8 \mathrm{kDa})$. Moreover, by using the SARMs example, negative USB-CBS ionization could be demonstrated as well, yielding less background interference and thus higher selectivity, lower LODs and improved overall quantitative performance. ${ }^{32}$ Aqueous solutions of ostarine (50 $\mu \mathrm{g} / \mathrm{L})$, andarine $(50 \mu \mathrm{g} / \mathrm{L}),[\mathrm{D}-\mathrm{Lys} 3]-G H R P-6(1 \mu \mathrm{g} / \mathrm{mL})$ and $\operatorname{rbST}(30 \mu \mathrm{g} / \mathrm{mL})$ were used and were sampled with Oasis HLB coated blades. Mass spectra were recorded in negative product-ion scan mode for ostarine and andarine (Figure $3 \mathrm{a}, \mathrm{b})$, positive ion scan and product-ion scan modes for [DLys3]-GHRP-6 (Figure 3c), and rbST (Figure 3d). Full scan spectra of [D-Lys3]-GHRP-6 and rbST demonstrated (multiple) protonated molecular ions. While [D-Lys3]-GHRP-6 was 
recorded as $[\mathrm{M}+2 \mathrm{H}]^{2+}$ at $m / z 465$, rbST displayed a charge state distribution which is in excellent agreement with direct analysis of rbST solutions.

Analysis of Sulfonamide Antibiotics in Milk Using USB-CBS-MS/MS. A method was developed and an initial single day validation was performed for sulfonamides in milk. Two individual MRM transitions were selected for each of the 13 antibiotics. Sulfonamide antibiotics were spiked at the maximum residue limit (MRL) concentration $(100 \mu \mathrm{g} / \mathrm{kg})$ in blank bovine milk. Clear MRM transitions were recorded for 12 out of the 13 sulfonamides spiked at MRL in bovine milk, exception being sulfacetamide (see Figure S3). The reconstructed MRM transitions corresponding to 9 sulfonamide antibiotics showed negligible background signals in blank milk matrix due to the selective extraction capabilities of the SPME coating on the blades and the additional selectivity of a tandem mass analyzer. ${ }^{31,33}$

Calibration curves with three replicates of 13 sulfonamide antibiotics and 9 corresponding internal standards were prepared in bovine milk. The matrix-matched calibration curves were used to determine the linearity and the LOD, the results are summarized in Table 1.

Table 1. Linearities and Established LODs of Semiquantitative USB-CBS-MS/MS Analysis of Sulfonamide Antibiotics in Bovine Milk Matrix

\begin{tabular}{|c|c|c|}
\hline analyte & $R^{2}$ & LOD $(\mu \mathrm{g} / \mathrm{kg})$ \\
\hline sulfamerazine & 0.975 & 25 \\
\hline sulfacetamide $^{a, b}$ & 0.438 & 200 \\
\hline sulfadiazine & 0.938 & 25 \\
\hline sulfathiazole & 0.937 & 25 \\
\hline sulfapyridine & 0.945 & 25 \\
\hline sulfamoxole $^{a}$ & 0.975 & 25 \\
\hline sulfadimidine & 0.930 & 25 \\
\hline sulfamethizole ${ }^{b}$ & 0.793 & 50 \\
\hline sulfamethoxypyridazine & 0.977 & 25 \\
\hline sulfachloropyridazine $^{a, b}$ & 0.572 & 200 \\
\hline sulfamethoxazole & 0.954 & 25 \\
\hline sulfaphenazole $e^{a, b}$ & 0.775 & 50 \\
\hline sulfaquinoxaline & 0.917 & 25 \\
\hline
\end{tabular}

${ }^{a}$ Response factor calculated using sulfamerazine- ${ }^{13} \mathrm{C}_{6}$ as internal standard. ${ }^{b}$ No significant discrimination of the response factor between the lowest concentration and respective blank bovine milk matrix.

For 11 out of 13 sulfonamide antibiotics, LODs below the MRL of $100 \mu \mathrm{g} / \mathrm{kg}$ milk were determined. Nine sulfonamide antibiotics had linearities of $R^{2} \geq 0.91$, indicating (semi-) quantitative performance of USB-CBS-MS/MS. As expected, less performance was achieved for sulfacetamide, sulfamethizole, sulfachloropyridazine and sulfaphenazole, since no isotope-labeled analogues were available as internal standards.

Of course, the eventual use of this method as an official (semi-)quantitative multiscreening tool will require a full validation, including precision, selectivity, specificity, and ruggedness. Nevertheless, these preliminary results for the linearities and LODs demonstrate the proof-of-concept and potential of USB-CBS-MS/MS as a very rapid semiquantitative analysis tool for multiple sulfonamide antibiotics in bovine milk.

\section{CONCLUSIONS}

A simplified inexpensive universal vendor-independent USBCBS ion source has been developed for direct MS analysis using low-cost components, in total less than $\$ 10$. The prototype ion source enabled versatile HV generation by OTG (micro-)USB devices like smartphones and tablets and could be positioned in front of different atmospheric pressure inlet mass spectrometers. The developed device demonstrated spray ionization in both positive and negative ionization mode and potential for a wide range of substances. The combination of USB-CBS with an outdated benchtop triple quadrupole mass spectrometer demonstrated the rapid (semi)quantitation of sulfonamide antibiotics in bovine milk. Note that existing legislation is under revision and direct MS approaches may be adopted. Suggested further improvements in the design of the USB-CBS ion source would comprise, for example, a single device for positive and negative ionization, more reproducible blade fixation and positioning, and automation to process more than one CBS device in parallel. Besides these design improvements, real field tests outside laboratory conditions should be performed.

This USB-CBS development will be compatible with any atmospheric inlet MS system and, together with future advances in fieldable tandem-MS analyzers, is expected to create many new options for efficient, inexpensive and direct on-site semiquantitative food analysis.

\section{ASSOCIATED CONTENT}

Supporting Information

The Supporting Information is available free of charge at https://pubs.acs.org/doi/10.1021/jasms.0c00307.

Additional data (Figures S1-S3 and Table S1) for the benchtop USB-CBS test (PDF)

Video showing Taylor cone formation and the associated spray jet (AVI)

\section{AUTHOR INFORMATION}

\section{Corresponding Author}

Marco H. Blokland - Wageningen Food Safety Research (WFSR), Part of Wageningen University \& Research, 6700 AE Wageningen, The Netherlands; $\odot$ orcid.org/0000-0002-37516065; Email: marco.blokland@wur.nl

\section{Authors}

Josha Jager - Wageningen Food Safety Research (WFSR), Part of Wageningen University \& Research, 6700 AE Wageningen, The Netherlands

Arjen Gerssen - Wageningen Food Safety Research (WFSR), Part of Wageningen University \& Research, 6700 AE Wageningen, The Netherlands

Janusz Pawliszyn - Department of Chemistry, University of Waterloo, Waterloo, Ontario N2L3G1, Canada; 이이.org/ 0000-0002-9975-5811

Saskia S. Sterk - Wageningen Food Safety Research (WFSR), Part of Wageningen University \& Research, 6700 AE Wageningen, The Netherlands

Michel W. F. Nielen - Wageningen Food Safety Research (WFSR), Part of Wageningen University \& Research, 6700 AE Wageningen, The Netherlands; Wageningen University, Laboratory of Organic Chemistry, 6708 WE Wageningen, The Netherlands; (1) orcid.org/0000-0003-4634-0249

Complete contact information is available at: 
https://pubs.acs.org/10.1021/jasms.0c00307

\section{Notes}

The authors declare no competing financial interest.

\section{ACKNOWLEDGMENTS}

Dr. German Gomez-Rios and Dr. Varoon Singh from the University of Waterloo are acknowledged for the production of coated blades. Jan Commandeur (MS Vision) is thanked for the gift of a dummy plug for the Waters Premier XE and technical support during oscilloscopic analysis.

\section{REFERENCES}

(1) Mielczarek, P.; Silberring, J.; Smoluch, M. Miniaturization in mass spectrometry. Mass Spectrom. Rev. 2020, 39, 453-470.

(2) Lawton, Z. E.; Traub, A.; Fatigante, W. L.; Mancias, J.; O'Leary, A. E.; Hall, S. E.; Wieland, J. R.; Oberacher, H.; Gizzi, M. C.; Mulligan, C. C. J. Analytical Validation of a Portable Mass Spectrometer Featuring Interchangeable, Ambient Ionization Sources for High Throughput Forensic Evidence Screening. J. Am. Soc. Mass Spectrom. 2017, 28, 1048-1059.

(3) Snyder, D. T.; Pulliam, C. J.; Ouyang, Z.; Cooks, R. G. Miniature and Fieldable Mass Spectrometers: Recent Advances. Anal. Chem. 2016, 88, 2-29.

(4) Zhang, X.; Zhang, H.; Yu, K.; Li, N.; Liu, Y.; Liu, X.; Zhang, H.; Yang, B.; Wu, W.; Gao, J.; Jiang, J. Rapid Monitoring Approach for Microplastics Using Portable Pyrolysis-Mass Spectrometry. Anal. Chem. 2020, 92, 4656-4662.

(5) Fiorentin, T. R.; Logan, B. K.; Martin, D. M.; Browne, T.; Rieders, E. F. Assessment of a portable quadrupole-based gas chromatography mass spectrometry for seized drug analysis. Forensic Sci. Int. 2020, 313, 110342.

(6) Wang, W.; Wang, S.; Xu, C.; Li, H.; Xing, Y.; Hou, K.; Li, H. Rapid Screening of Trace Volatile and Nonvolatile Illegal Drugs by Miniature Ion Trap Mass Spectrometry: Synchronized FlashThermal-Desorption Purging and Ion Injection. Anal. Chem. 2019, 91, 10212-10220.

(7) Zhou, C.; Wu, H.; Zhang, X.; Zhang, Y.; Xie, W.; Xu, W. Anal. Chem. 2019, 91, 8808-8813.

(8) Chen, H.; Chen, C.; Huang, W.; Li, M.; Xiao, Y.; Jiang, D.; Li, H. High-Throughput and Direct Sample Screening Using a Laser Spray Ionization Miniature Mass Spectrometer. Anal. Chem. 2019, 91, 9138-9146.

(9) Fedick, P. W.; Pu, F.; Morato, N. M.; Cooks, R. G. Identification and Confirmation of Fentanyls on Paper using Portable Surface Enhanced Raman Spectroscopy and Paper Spray Ionization Mass Spectrometry. J. Am. Soc. Mass Spectrom. 2020, 31, 735-741.

(10) Pulliam, C. J.; Bain, R. M.; Osswald, H. L.; Snyder, D. T.; Fedick, P. W.; Ayrton, S. T.; Flick, T. G.; Cooks, R. G. Simultaneous Online Monitoring of Multiple Reactions Using a Miniature Mass Spectrometer. Anal. Chem. 2017, 89, 6969-6975.

(11) Wang, S.; Wang, W.; Li, H.; Xing, Y.; Hou, K.; Li, H. Rapid OnSite Detection of Illegal Drugs in Complex Matrix by Thermal Desorption Acetone-Assisted Photoionization Miniature Ion Trap Mass Spectrometer. Anal. Chem. 2019, 91, 3845-3851.

(12) Blokland, M. H.; Gerssen, A.; Zoontjes, P. W.; Pawliszyn, J.; Nielen, M. W. F. Potential of recent ambient ionization techniques for future food contaminant analysis using (trans) portable mass spectrometry. Food Anal. Methods 2020, 13, 706-717.

(13) Klampfl, C. W.; Himmelsbach, M. Direct ionization methods in mass spectrometry: An overview. Anal. Chim. Acta 2015, 890, 44-59.

(14) Huang, M.-Z.; Cheng, S.-C.; Cho, Y.-T.; Shiea, J. Ambient ionization mass spectrometry: a tutorial. Anal. Chim. Acta 2011, 702, $1-15$.

(15) Gómez-Ríos, G. A.; Pawliszyn, J. Development of coated blade spray ionization mass spectrometry for the quantitation of target analytes present in complex matrices. Angew. Chem., Int. Ed. 2014, 53, 14503-14507.
(16) Khaled, A.; Gómez-Ríos, G. A.; Pawliszyn, J. Optimization of Coated Blade Spray for Rapid Screening and Quantitation of 105 Veterinary Drugs in Biological Tissue Samples. Anal. Chem. 2020, 92, 5937-5943.

(17) Souza-Silva, É. A.; Jiang, R.; Rodríguez-Lafuente, A.; Gionfriddo, E.; Pawliszyn, J. A critical review of the state of the art of solid-phase microextraction of complex matrices I. Environmental analysis. TrAC, Trends Anal. Chem. 2015, 71, 224-235.

(18) Kasperkiewicz, A.; Pawliszyn, J. Multiresidue pesticide quantitation in multiple fruit matrices via automated coated blade spray and liquid chromatography coupled to triple quadrupole mass spectrometry. Food Chem. 2021, 339, 127815.

(19) Blokland, M. H.; Gerssen, A.; Jager, J.; Nielen, M. W. F. Ionization source, functional combination with a mobile device and arrangement with an atmospheric inlet mass spectrometry system Eur. Pat. Appl. 201775749, 2020.

(20) Ross, G. M. S.; Bremer, M. G. E. G.; Nielen, M. W. F. Consumer-friendly food allergen detection: moving towards smartphone-based immunoassays. Anal. Bioanal. Chem. 2018, 410, 53535371.

(21) Devereaux, Z. J.; Reynolds, C. A.; Fischer, J. L.; Foley, C. D.; DeLeeuw, J. L.; Wager-Miller, J.; Narayan, S. B.; Mackie, K.; Trimpin, S. Matrix-Assisted Ionization on a Portable Mass Spectrometer: Analysis Directly from Biological and Synthetic Materials. Anal. Chem. 2016, 88, 10831-10836.

(22) Tascon, M.; Gómez-Ríos, G. A.; Reyes-Garcés, N.; Poole, J.; Boyac1, E.; Pawliszyn, J. Ultra-fast quantitation of voriconazole in human plasma by coated blade spray mass spectrometry. J. Pharm. Biomed. Anal. 2017, 144, 106-111.

(23) Piri-Moghadam, H.; Ahmadi, F.; Gómez-Ríos, G. A.; Boyacı, E.; Reyes-Garcés, N.; Aghakhani, A.; Bojko, B.; Pawliszyn, J. Fast Quantitation of Target Analytes in Small Volumes of Complex Samples by Matrix-Compatible Solid-Phase Microextraction Devices. Angew. Chem., Int. Ed. 2016, 55, 7510-7514.

(24) Liu, J.; Wang, H.; Manicke, N. E.; Lin, J.-M.; Cooks, R. G.; Ouyang, Z. Development, characterization, and application of paper spray ionization. Anal. Chem. 2010, 82, 2463-2471.

(25) Teunissen, S. F.; Fedick, P. W.; Berendsen, B. J. A.; Nielen, M. W. F.; Eberlin, M. N.; Graham Cooks, R.; van Asten, A. C. Novel Selectivity-Based Forensic Toxicological Validation of a Paper Spray Mass Spectrometry Method for the Quantitative Determination of Eight Amphetamines in Whole Blood. J. Am. Soc. Mass Spectrom. 2017, 28, 2665-2676.

(26) Aresta, A.; Calvano, C. D.; Palmisano, F.; Zambonin, C. G. Determination of clenbuterol in human urine and serum by solidphase microextraction coupled to liquid chromatography. J. Pharm. Biomed. Anal. 2008, 47, 641-645.

(27) Parr, M. K.; Blokland, M. H.; Liebetrau, F.; Schmidt, A. H.; Meijer, T.; Stanic, M.; Kwiatkowska, D.; Waraksa, E.; Sterk, S. S. Distinction of clenbuterol intake from drug or contaminated food of animal origin in a controlled administration trial - the potential of enantiomeric separation for doping control analysis. Food Addit. Contam., Part A 2017, 34, 525-535.

(28) Carter, W. J.; Dang, A. Q.; Faas, F. H.; Lynch, M. E. Effects of clenbuterol on skeletal muscle mass, body composition, and recovery from surgical stress in senescent rats. Metab., Clin. Exp. 1991, 40, $855-860$.

(29) Nielen, M. W. F.; Lasaroms, J. J. P.; Essers, M. L.; Oosterink, J. E.; Meijer, T.; Sanders, M. B.; Zuidema, T.; Stolker, A. A. M. Multiresidue analysis of beta-agonists in bovine and porcine urine, feed and hair using liquid chromatography electrospray ionisation tandem mass spectrometry. Anal. Bioanal. Chem. 2008, 391, 199-210.

(30) Blanca, J.; Muñoz, P.; Morgado, M.; Méndez, N.; Aranda, A.; Reuvers, T.; Hooghuis, H. A Portable Photoelectric Sensor Based on Colloidal Gold Immunochromatographic Strips for Rapid Determination of Clenbuterol in Pig Urine. Anal. Chim. Acta 2005, 529, 199205. 
(31) Berendsen, B. J. A.; Stolker, L. A. M.; Nielen, M. W. F. The (un)certainty of selectivity in liquid chromatography tandem mass spectrometry. J. Am. Soc. Mass Spectrom. 2013, 24, 154-163.

(32) Chiu, F. C. K.; Lo, C. M. Y. Observation of amide anions in solution by electrospray ionization mass spectrometry. J. Am. Soc. Mass Spectrom. 2000, 11, 1061-1064.

(33) Rickert, D. A.; Gómez-Ríos, G. A.; Nazdrajić, E.; Tascon, M.; Kulasingam, V.; Pawliszyn, J. Evaluation of a coated blade spraytandem mass spectrometry assay as a new tool for the determination of immunosuppressive drugs in whole blood. Anal. Bioanal. Chem. 2020, 412, 5067-5076. 